\title{
Pharmacological and Predicted Activities of Natural Azo Compounds
}

\author{
Valery M. Dembitsky • Tatyana A. Gloriozova • \\ Vladimir V. Poroikov
}

Received: 14 September 2016/ Accepted: 26 December 2016/Published online: 4 January 2017

(C) The Author(s) 2017. This article is published with open access at Springerlink.com

\begin{abstract}
This paper describes research on natural azo compounds isolated from fungi, plant, bacteria, and invertebrates. More than 120 biologically active diazene containing alkaloids demonstrate confirmed pharmacological activity, including antitumor, antimicrobial, and antibacterial effects. The structures, origin, and biological activities of azo compounds are reviewed. Utilizing the computer program PASS, some structure-activity relationship new activities are also predicted, pointing toward possible new applications of these compounds. This article emphasizes the role of natural azo compounds as an important source of drug prototypes and leads for drug discovery.
\end{abstract}

Keywords Azo metabolites $\cdot$ Alkaloids $\cdot$ Fungi $\cdot$ Plant $\cdot$ Bacteria $\cdot$ Sponges $\cdot$ SAR

\section{Introduction}

Natural azo compounds are diazene containing compounds. Also called diimine or diimide, these metabolites have an azo moiety $(-\mathrm{N}=\mathrm{N}-)$ [1-4]. The majority of natural diazene alkaloids have been isolated from microorganisms, plant parts (bark, berries, leaves, roots, and wood), fungi, fungal endophytes, lichenized ascomycetes and marine invertebrates [5-18].

Using the structure-activity relationships (SAR) approach realized in the computer program PASS, some additional activities were also predicted, indicating possible new applications for these compounds. Keeping in mind that presented below data on biological activity of azo metabolites characterize only a small part of possible biological potential in these molecules, we tried to estimate their biological activity spectra by computer prediction.

V. M. Dembitsky $(\bowtie)$

National Scientific Center of Marine Biology, 17 Palchevsky

Str., Vladivostok, Russia 690041

e-mail: valery@dembitsky.com

T. A. Gloriozova · V. V. Poroikov

Institute of Biomedical Chemistry, Moscow, Russia 119121
For this purpose we used computer program PASS [19, 20], which predicts more than 7000 pharmacological activities, mechanisms of action, mutagenicity, carcinogenicity, teratogenicity and embryotoxicity on the basis of structural formulae of compounds. PASS predictions are based on SAR analysis of the training set consisting of more than 900000 of drugs, drug-candidates and lead compounds. Algorithm of PASS predictions is described in detail in several publications [21-24]. Using MOL or SD files as an input for PASS program, user may get a list of probable biological activities for any drug-like molecule as an output. For each activity $\mathrm{Pa}$ and $\mathrm{Pi}$ values are calculated, which can be interpreted either as the probabilities of a molecule belonging to the classes of active and inactive compounds respectively, or as the probabilities of the first and second kind of errors in prediction. Although the majority of the known biological activities for respective azo compounds are associated with antineoplastic action, their number is less than $60 \%$ among the predicted focal activities. A computer analysis of the predicted biological activity spectra showed that 58 types of biological activity are predicted with $\mathrm{Pa}>70 \%, 199$ with $\mathrm{Pa}>50 \%, 463$ with $\mathrm{Pa}>30 \%$, and 810 with $\mathrm{Pa}>\mathrm{Pi}$. This paper 
emphasizes the role of natural azo dyes as important sources for drug discovery.

\section{Azo Metabolites Derived from Actinomycetes and Fungal Species}

Valanimycin (1), an azoxy antibiotic, was isolated from culture broths of Streptomyces viridifaciens MG456-hF10. It was active against both Gram-positive and Gram-negative bacteria, especially against $E$. coli BE1121, a DNA repair deficient mutant of $E$. coli $\mathrm{K} 12$. Valanimycin was toxic to in vitro cultures of cells of mouse leukemia L1210, $\mathrm{P} 388 / \mathrm{S}$, and P388/ADR, with $\mathrm{IC}_{50}$ values of $0.8,2.7$, and $1.4 \mathrm{pg} / \mathrm{mL}$, respectively. It prolonged the life span of mice inoculated with Ehrlich carcinoma or L1210 [25]. Valanimycin derivative (2) was found in culture broth of a $S$. viridifaciens MG456-hF10 during biosynthesis of valanimycin (1) [26], and the elucidation of the structure was carried out on the more stable ammonia adduct (3) [27]. Predicted activities compounds (1-10) shown in Table 1 and structures shown in Fig. 1. $\alpha, \beta$-Unsaturated azoxycontaining antibiotic LL-BH872 $\alpha$ (4) was isolated from Streptomyces hinnulinis [28]. More recently, LL-BH872a, 2(Z)-OH (5) produced by Actinomadura sp., was isolated from the roots of Prunus armeniaca [27], and antibiotic LL-BH872a, 2(Z)-OH, 4'(Z)-OH (6), produced by Streptomyces misionensis [29].

Two antifungal antibiotics, maniwamycins A (7) and B (8), were isolated from the culture broth of Streptomyces prasinopilosus. Both antibiotics showed broad antifungal activities against Candida albicans IFM 40001, C. albicans N 508, C. albicans TIMM 0228, C. albicans TIMM 0237, Cryptococcus neoformans IFM 40038, Nannizzia otae JCM

Table 1 Confirmed and new biological activities of azo compounds (1-10) derived from actinomycetes

\begin{tabular}{|c|c|c|c|}
\hline No. & Activity reviewed & Activities confirmed $(\mathrm{Pa})$ & Additional predicted activities $\left(\mathrm{Pa}^{\mathrm{a}}\right)$ \\
\hline \multirow[t]{2}{*}{1} & \multirow[t]{2}{*}{ Antibiotic antineoplastic } & Antineoplastic (0.985) & Phobic disorders treatment (0.819) \\
\hline & & Antineoplastic antibiotic $(0.848)$ & Hepatic disorders treatment $(0.662)$ \\
\hline \multirow[t]{3}{*}{2} & \multirow[t]{3}{*}{ Not studied } & \multirow[t]{3}{*}{-} & Antineoplastic (0.880) \\
\hline & & & Phobic disorders treatment $(0.864)$ \\
\hline & & & Hepatic disorders treatment $(0.852)$ \\
\hline \multirow[t]{3}{*}{3} & \multirow[t]{3}{*}{ Not studied } & \multirow[t]{3}{*}{-} & Phobic disorders treatment $(0.907)$ \\
\hline & & & Antiseborrheic (0.861) \\
\hline & & & Antineoplastic (0.862) \\
\hline \multirow[t]{3}{*}{4} & \multirow[t]{3}{*}{ Antibiotic } & \multirow[t]{3}{*}{-} & Hepatic disorders treatment $(0.872)$ \\
\hline & & & Antineoplastic (0.736) \\
\hline & & & Antieczematic (0.733) \\
\hline \multirow[t]{3}{*}{5} & \multirow[t]{3}{*}{ Antibiotic } & \multirow[t]{3}{*}{ Antibacterial (0.507) } & Hepatic disorders treatment (0.819) \\
\hline & & & Antiviral (arbovirus) (0.783) \\
\hline & & & Antineoplastic (0.746) \\
\hline \multirow[t]{3}{*}{6} & \multirow[t]{3}{*}{ Antibiotic } & \multirow[t]{3}{*}{ Antibacterial (0.527) } & Hepatic disorders treatment $(0.765)$ \\
\hline & & & Antineoplastic (0.760) \\
\hline & & & Antifungal (0.653) \\
\hline \multirow[t]{3}{*}{7} & \multirow[t]{3}{*}{ Antibiotic antifungal } & \multirow[t]{3}{*}{ Antifungal (0.640) } & Hepatic disorders treatment $(0.778)$ \\
\hline & & & Antineoplastic (0.763) \\
\hline & & & Antieczematic (0.717) \\
\hline \multirow[t]{3}{*}{8} & \multirow[t]{3}{*}{ Antibiotic antifungal } & \multirow[t]{3}{*}{-} & Phobic disorders treatment $(0.860)$ \\
\hline & & & Mucositis treatment $(0.765)$ \\
\hline & & & Antiviral (arbovirus) (0.747) \\
\hline \multirow[t]{3}{*}{9} & \multirow[t]{3}{*}{ Antifungal } & \multirow[t]{3}{*}{ Antifungal (0.658) } & Hepatic disorders treatment $(0.793)$ \\
\hline & & & Antiviral (arbovirus) (0.771) \\
\hline & & & Antineoplastic (0.779) \\
\hline \multirow[t]{3}{*}{10} & \multirow[t]{3}{*}{ Antifungal } & \multirow[t]{3}{*}{ Antifungal (0.632) } & Hepatic disorders treatment (0.819) \\
\hline & & & Antiviral (arbovirus) (0.783) \\
\hline & & & Antineoplastic (0.746) \\
\hline
\end{tabular}

a Only activities with $\mathrm{Pa}>0.5$ are shown 


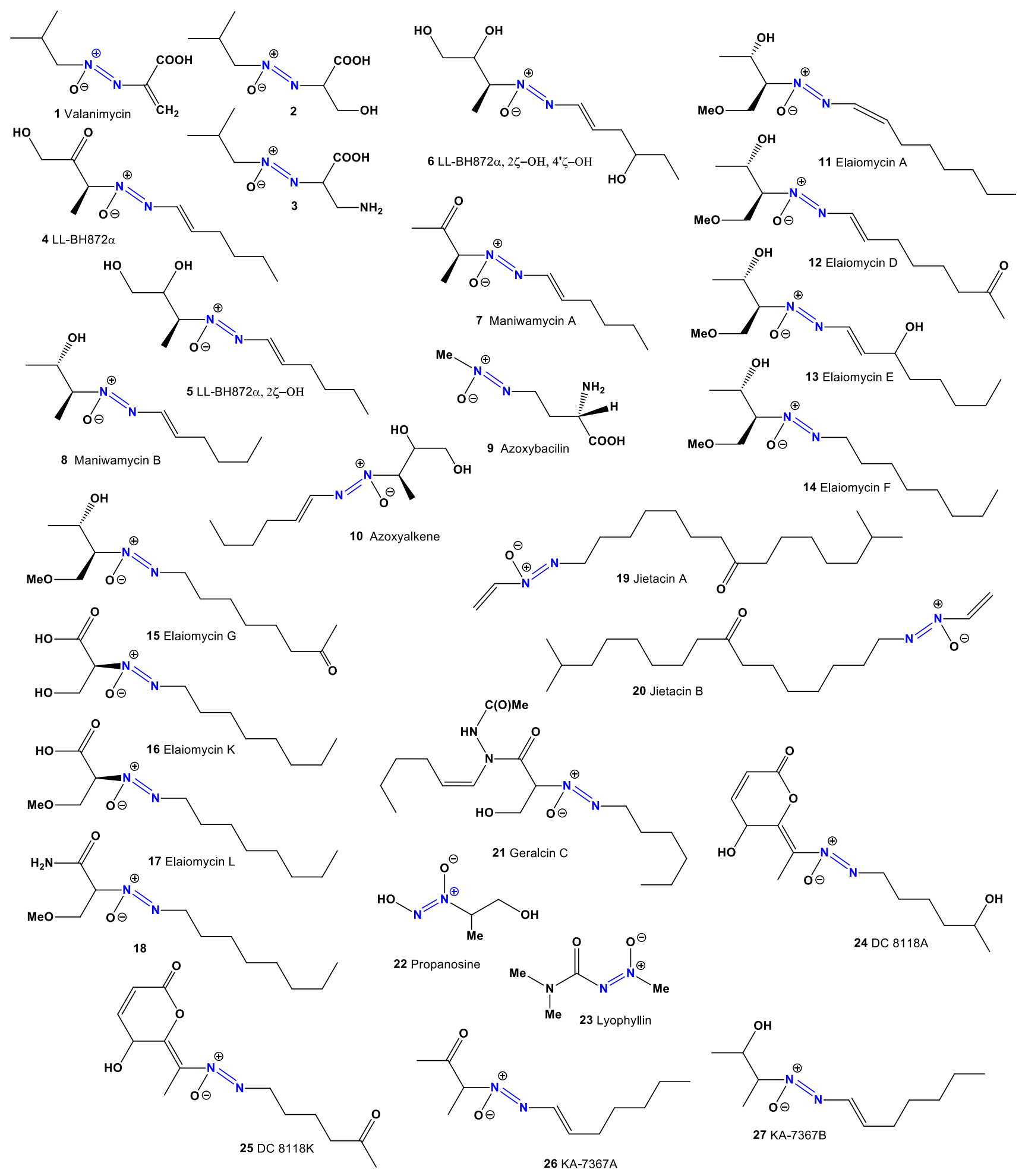

Fig. 1 Biological active azo compounds derived from actinomycetes

1909, Trichophyton mentagrophytes IFM 40769, T. mentagrophytes IFM 40771, T. rubrum IFM 40768, and Staphylococcus aureus FDA 209P [30].

The microbial antifungal agent azoxybacilin (9) was isolated from the culture broth of Bacillus cereus NR2991.
Azoxybacilin exhibits broad spectrum antifungal activity, especially against mycelial fungi, such as Aspergillus fumigatus and Trichophyton mentagrophytes [31, 32]. Azoxyalkene (10) is an unstable azoxy compound isolated from Actinomadura sp., an actinomycete growing in 
Table 2 Confirmed and new biological activities of azo compounds (11-21) derived from actinomycetes

\begin{tabular}{|c|c|c|c|}
\hline No. & Activity reviewed & Activities confirmed $(\mathrm{Pa})$ & Additional predicted activities $\left(\mathrm{Pa}^{\mathrm{a}}\right)$ \\
\hline 11 & Antibiotic anti-mycobacterial & Antibacterial (0.489) & $\begin{array}{l}\text { Hepatic disorders treatment }(0.733) \\
\text { Antineoplastic }(0.731) \\
\text { Antieczematic }(0.697)\end{array}$ \\
\hline 12 & Antibiotic antifungal cytotoxic & $\begin{array}{l}\text { Antifungal }(0.646) \\
\text { Antineoplastic (0.738) }\end{array}$ & $\begin{array}{l}\text { Hepatic disorders treatment }(0.763) \\
\text { Vasodilator, peripheral }(0.738)\end{array}$ \\
\hline 13 & Antibiotic antifungal cytotoxic & Antifungal (0.639) & $\begin{array}{l}\text { Vasodilator }(0.722) \\
\text { Antiinfective }(0.684) \\
\text { Vasodilator }(0.637)\end{array}$ \\
\hline 14 & Antibiotic cytotoxic & Antineoplastic (0.549) & $\begin{array}{l}\text { Phobic disorders treatment }(0.769) \\
\text { Antiviral (arbovirus) }(0.688) \\
\text { Natural killer cell stimulant }(0.637)\end{array}$ \\
\hline 15 & Antibiotic cytotoxic & Antineoplastic (0.599) & $\begin{array}{l}\text { Hepatic disorders treatment }(0.604) \\
\text { Vasodilator, peripheral }(0.609)\end{array}$ \\
\hline 16 & Antibiotic antibacterial & - & $\begin{array}{l}\text { Phobic disorders treatment }(0.834) \\
\text { Antiviral (arbovirus) }(0.805) \\
\text { Mucositis treatment }(0.754)\end{array}$ \\
\hline 17 & Antibiotic antibacterial & - & $\begin{array}{l}\text { Phobic disorders treatment }(0.888) \\
\text { Preneoplastic }(0.779) \\
\text { Mucositis treatment }(0.756)\end{array}$ \\
\hline 18 & Antibiotic antibacterial & - & $\begin{array}{l}\text { Phobic disorders treatment }(0.837) \\
\text { Mucositis treatment }(0.734) \\
\text { Natural killer cell stimulant }(0.668)\end{array}$ \\
\hline 19 & Nematocide & - & $\begin{array}{l}\text { Hepatic disorders treatment }(0.849) \\
\text { Phobic disorders treatment }(0.672) \\
\text { Antifungal }(0.569)\end{array}$ \\
\hline 20 & Nematocide & - & $\begin{array}{l}\text { Hepatic disorders treatment }(0.849) \\
\text { Phobic disorders treatment }(0.672) \\
\text { Antifungal }(0.569)\end{array}$ \\
\hline 21 & Antibiotic antineoplastic & Antineoplastic (0.672) & Antiviral (arbovirus) (0.557) \\
\hline
\end{tabular}

${ }^{a}$ Only activities with $\mathrm{Pa}>0.5$ are shown

Table 3 Confirmed and new biological activities of azo compounds (22-39) derived from actinomycetes

\begin{tabular}{llll}
\hline No. & Activity reviewed & Activities confirmed $(\mathrm{Pa})$ & Additional predicted activities $\left(\mathrm{Pa}^{\mathrm{a}}\right)$ \\
\hline $\mathbf{2 2}$ & Antibiotic antifungal & - & Antineoplastic $(0.845)$ \\
& & & Phobic disorders treatment $(0.775)$ \\
& & Antiviral (picornavirus) $(0.735)$ \\
$\mathbf{2 3}$ & Antibiotic & - & Antineoplastic $(0.781)$ \\
& & & Phobic disorders treatment $(0.750)$ \\
& & & Antiviral (arbovirus) $(0.565)$ \\
$\mathbf{2 4}$ & Antibacterial antineoplastic & Antineoplastic $(0.923)$ & Antifungal $(0.633)$ \\
& & Antibacterial $(0.613)$ & Genital warts treatment $(0.648)$ \\
$\mathbf{2 5}$ & Antibacterial antineoplastic & Antineoplastic $(0.927)$ & Spasmolytic, urinary $(0.605)$ \\
& & Antibacterial $(0.573)$ & Spasmolytic, urinary $(0.687)$ \\
& & Genital warts treatment $(0.648)$
\end{tabular}


Table 3 continued

\begin{tabular}{|c|c|c|c|}
\hline No. & Activity reviewed & Activities confirmed $(\mathrm{Pa})$ & Additional predicted activities $\left(\mathrm{Pa}^{\mathrm{a}}\right)$ \\
\hline 26 & Antibiotic antifungal & $\begin{array}{l}\text { Antifungal }(0.640) \\
\text { Antibacterial }(0.474)\end{array}$ & $\begin{array}{l}\text { Hepatic disorders treatment }(0.778) \\
\text { Antineoplastic }(0.763) \\
\text { Antieczematic }(0.717)\end{array}$ \\
\hline 27 & Antibiotic antifungal & $\begin{array}{l}\text { Antifungal }(0.658) \\
\text { Antibacterial }(0.514)\end{array}$ & $\begin{array}{l}\text { Hepatic disorders treatment }(0.793) \\
\text { Antiviral (arbovirus) }(0.771) \\
\text { Antineoplastic }(0.779)\end{array}$ \\
\hline 28 & $\begin{array}{l}\text { Antineoplastic antibiotic cytotoxic } \\
\text { ornithine decarboxylase inhibitor }\end{array}$ & Anti-Helicobacter pylori (0.995) & $\begin{array}{l}\text { Kidney function stimulant }(0.636) \\
\text { Antieczematic }(0.644) \\
\text { Preneoplastic conditions treatment }(0.590)\end{array}$ \\
\hline 29 & Antineoplastic antibiotic & - & $\begin{array}{l}\text { Anti-Helicobacter pylori }(0.994) \\
\text { Preneoplastic conditions treatment }(0.513)\end{array}$ \\
\hline 30 & Microtubule inhibitor & - & $\begin{array}{l}\text { Anti-Helicobacter pylori }(0.893) \\
\text { Antieczematic }(0.751) \\
\text { Fibrinolytic }(0.638)\end{array}$ \\
\hline 31 & Microtubule inhibitor & - & $\begin{array}{l}\text { Anti-Helicobacter pylori }(0.915) \\
\text { Preneoplastic conditions treatment }(0.670) \\
\text { Kidney function stimulant }(0.654)\end{array}$ \\
\hline 32 & Not studied & - & $\begin{array}{l}\text { Antiseborrheic }(0.793) \\
\text { Antiinflammatory }(0.780) \\
\text { Hemostatic }(0.668)\end{array}$ \\
\hline 33 & Nematocide & - & $\begin{array}{l}\text { Antiseborrheic }(0.793) \\
\text { Antiinflammatory }(0.780) \\
\text { Hemostatic }(0.668)\end{array}$ \\
\hline 34 & Nematocide & - & $\begin{array}{l}\text { Anti-Helicobacter pylori }(0.939) \\
\text { Antiseborrheic }(0.717) \\
\text { Alopecia treatment }(0.650)\end{array}$ \\
\hline 35 & Not studied & - & $\begin{array}{l}\text { Antiinflammatory }(0.714) \\
\text { Phobic disorders treatment }(0.682) \\
\text { Preneoplastic conditions treatment }(0.617)\end{array}$ \\
\hline 36 & Not studied & - & $\begin{array}{l}\text { Anti-Helicobacter pylori }(0.932) \\
\text { Preneoplastic conditions treatment }(0.605)\end{array}$ \\
\hline 37 & Nematocide cytotoxic & - & $\begin{array}{l}\text { Anti-Helicobacter pylori }(0.932) \\
\text { Phobic disorders treatment }(0.672) \\
\text { Preneoplastic conditions treatment }(0.568)\end{array}$ \\
\hline 38 & Not studied & - & $\begin{array}{l}\text { Apoptosis agonist }(0.935) \\
\text { Antineoplastic }(0.788) \\
\text { Alopecia treatment }(0.653)\end{array}$ \\
\hline 39 & Not studied & - & $\begin{array}{l}\text { Apoptosis agonist }(0.920) \\
\text { Antineoplastic }(0.724) \\
\text { Antiviral (arbovirus) (0.622) }\end{array}$ \\
\hline
\end{tabular}

${ }^{a}$ Only activities with $\mathrm{Pa}>0.5$ are shown

apricot roots. Preliminary biological assays revealed that exhibits weak antifungal activity against Rhodotorula sp. [27].

Elaiomycin (11) is an azoxy antibiotic that was first isolated from Streptomyces hepaticus and found to strongly inhibit the growth of Mycobacterium tuberculosis [33-36].
Elaiomycins D-G (12-15), antimicrobial and cytotoxic azoxides, were isolated from Streptomyces sp. HKI0708. Individual elaiomycins exhibit specific antimycobacterial, anti-Aspergillus, and cytotoxic activities, providing provisional data on SAR [37, 38]. Predicted activities compounds (11-21) shown in Table 2 and the structures shown 
Fig. 2 Aromatic azo compounds derived from actinomycetes and fungal species

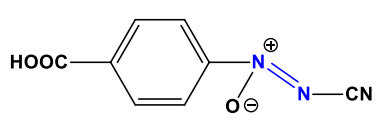

28 Calvatic acid

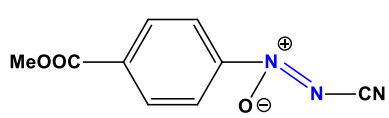

29 Calvatic acid, Methyl ester<smiles>CCOC(=O)N=[N+]([O-])c1ccc(C(=O)O)cc1</smiles>

30<smiles>NC(=O)N=[N+]([O-])c1ccc(C(=O)O)cc1</smiles>

31<smiles>CSC1=CC(=O)C=C(S(C)(=O)=O)/C1=N\NC(N)=O</smiles>

40 Deoxyrubroflavin<smiles>CS(=O)c1cc(O)cc(S(C)=O)c1/N=N/C(N)=O</smiles><smiles>CS(=O)c1cc(O)cc(S(C)=O)c1/N=N/C(N)=O</smiles><smiles>CS(=O)(=O)c1cc(O)cc(S(C)(=O)=O)c1/N=N/C(N)=O</smiles>

RO

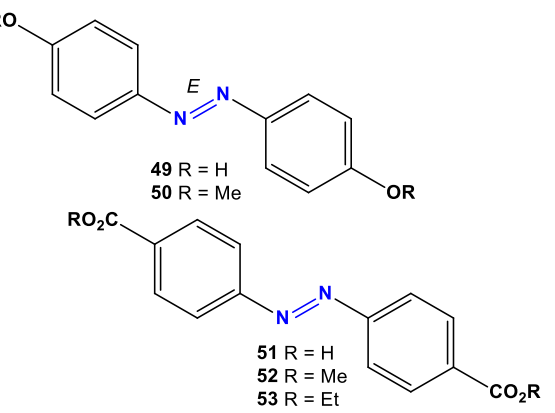<smiles>COc1ccc(/N=N/C(N)=O)cc1</smiles><smiles>COc1ccc(NC(=O)/N=N/C(N)=O)cc1</smiles><smiles>O=C(O)c1ccc(/N=[N+](\[O-])c2ccc(F)cc2)cc1</smiles>

46<smiles>Cc1ccc([N+]([O-])=Nc2ccc(CO)cc2)cc1</smiles><smiles>O=C(O)c1ccc(N=N/C(=N\Nc2ccc(C(=O)O)cc2)c2ccccc2)cc1</smiles>

47

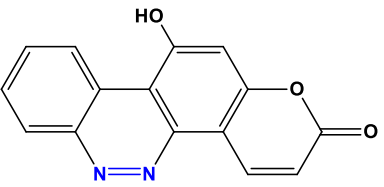

48 Necatorin<smiles>[R]c1ccc(/N=N/c2ccc(C)cc2)cc1</smiles>

$\mathrm{RO}_{2} \mathrm{C}$

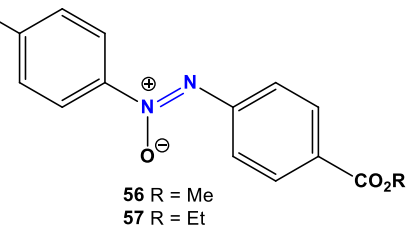


Table 4 Confirmed and new biological activities of azo compounds (40-58) derived from actinomycetes and fungal species

\begin{tabular}{|c|c|c|c|}
\hline No. & Activity reviewed & Activities confirmed $(\mathrm{Pa})$ & Additional predicted activities $\left(\mathrm{Pa}^{\mathrm{a}}\right)$ \\
\hline 40 & Not studied & - & $\begin{array}{l}\text { Antiinflammatory }(0.957) \\
\text { Antineoplastic }(0.837) \\
\text { Hemostatic }(0.822)\end{array}$ \\
\hline 41 & Not studied & - & $\begin{array}{l}\text { Hemostatic }(0.962) \\
\text { Antiinflammatory }(0.956) \\
\text { Antineoplastic }(0.808)\end{array}$ \\
\hline 42 & Not studied & - & $\begin{array}{l}\text { Antiinflammatory }(0.964) \\
\text { Hemostatic }(0.952) \\
\text { Antineoplastic }(0.833)\end{array}$ \\
\hline 43 & Not studied & - & $\begin{array}{l}\text { Antiinflammatory }(0.953) \\
\text { Antineoplastic }(0.772) \\
\text { Hemostatic }(0.577)\end{array}$ \\
\hline 44 & Not studied & - & $\begin{array}{l}\text { Antiinflammatory }(0.961) \\
\text { Antiarthritic }(0.849) \\
\text { Antineoplastic }(0.781)\end{array}$ \\
\hline 45 & Insecticide & - & $\begin{array}{l}\text { Antieczematic }(0.732) \\
\text { Kidney function stimulant }(0.701) \\
\text { Preneoplastic conditions }(0.677)\end{array}$ \\
\hline 46 & Insecticide & - & $\begin{array}{l}\text { Immunosuppressant }(0.647) \\
\text { Antieczematic }(0.659) \\
\text { Fibrinolytic }(0.606)\end{array}$ \\
\hline 47 & Not studied & - & $\begin{array}{l}\text { Preneoplastic }(0.692) \\
\text { Kidney function stimulant }(0.631)\end{array}$ \\
\hline 48 & Not studied & - & $\begin{array}{l}\text { Antimutagenic }(0.758) \\
\text { Spasmolytic, urinary }(0.699) \\
\text { Antineoplastic }(0.660)\end{array}$ \\
\hline 49 & Not studied & - & $\begin{array}{l}\text { Antiseborrheic }(0.874) \\
\text { Phobic disorders treatment }(0.784) \\
\text { Kidney function stimulant }(0.752)\end{array}$ \\
\hline 50 & Not studied & - & $\begin{array}{l}\text { Carminative }(0.817) \\
\text { Phobic disorders treatment }(0.786) \\
\text { Antiseborrheic }(0.763)\end{array}$ \\
\hline 51 & Not studied & - & $\begin{array}{l}\text { Antieczematic }(0.843) \\
\text { Phobic disorders treatment }(0.832) \\
\text { Kidney function stimulant }(0.786)\end{array}$ \\
\hline 52 & Not studied & - & $\begin{array}{l}\text { Phobic disorders treatment }(0.840) \\
\text { Fibrinolytic }(0.720) \\
\text { Preneoplastic conditions }(0.703)\end{array}$ \\
\hline 53 & Not studied & - & $\begin{array}{l}\text { Acaricide }(0.821) \\
\text { Phobic disorders treatment }(0.782) \\
\text { Antiseborrheic }(0.773)\end{array}$ \\
\hline 54 & Not studied & - & $\begin{array}{l}\text { Alopecia treatment }(0.762) \\
\text { Phobic disorders treatment }(0.777) \\
\text { Antiinflammatory, intestinal }(0.699)\end{array}$ \\
\hline 55 & Not studied & - & $\begin{array}{l}\text { Phobic disorders treatment }(0.916) \\
\text { Antiseborrheic }(0.802) \\
\text { Acaricide }(0.726)\end{array}$ \\
\hline
\end{tabular}


Table 4 continued

\begin{tabular}{llll}
\hline No. & Activity reviewed & Activities confirmed $(\mathrm{Pa})$ & Additional predicted activities $\left(\mathrm{Pa}^{\mathrm{a}}\right)$ \\
\hline $\mathbf{5 6}$ & Not studied & - & Phobic disorders treatment $(0.700)$ \\
& & & Preneoplastic conditions treatment $(0.606)$ \\
& & Immunosuppressant $(0.612)$ \\
$\mathbf{5 7}$ & Not studied & - & Fibrinolytic $(0.640)$ \\
& & - & Acaricide $(0.636)$ \\
$\mathbf{5 8}$ & Not studied & & Mucositis treatment $(0.831)$ \\
& & Immunosuppressant $(0.690)$ \\
\hline
\end{tabular}

${ }^{a}$ Only activities with $\mathrm{Pa}>0.5$ are shown

Table 5 Confirmed and new biological activities of azo compounds (59-77) derived from actinomycetes and fungal species

\begin{tabular}{|c|c|c|c|}
\hline No. & Activity reviewed & Activities confirmed $(\mathrm{Pa})$ & Additional predicted activities $\left(\mathrm{Pa}^{\mathrm{a}}\right)$ \\
\hline 59 & Not studied & - & $\begin{array}{l}\text { Mucositis treatment }(0.776) \\
\text { Immunosuppressant }(0.691)\end{array}$ \\
\hline 60 & Not studied & - & $\begin{array}{l}\text { Antiviral (arbovirus) } \\
(0.694) \\
\text { Immunosuppressant }(0.693) \\
\text { Antipsoriatic }(0.625)\end{array}$ \\
\hline 61 & Not studied & - & $\begin{array}{l}\text { Genital warts treatment }(0.726) \\
\text { Antineoplastic }(0.691)\end{array}$ \\
\hline 62 & Not studied & - & $\begin{array}{l}\text { Genital warts treatment }(0.726) \\
\text { Antineoplastic }(0.704) \\
\text { Antiinflammatory }(0.625)\end{array}$ \\
\hline 63 & Not studied & - & $\begin{array}{l}\text { Genital warts treatment }(0.726) \\
\text { Antileukemic }(0.567)\end{array}$ \\
\hline 64 & Not studied & - & $\begin{array}{l}\text { Mucositis treatment }(0.761) \\
\text { Antiviral (arbovirus) }(0.744)\end{array}$ \\
\hline 65 & Antibiotic antineoplastic & Antineoplastic (breast cancer) (0.552) & $\begin{array}{l}\text { Alopecia treatment }(0.641) \\
\text { Vascular (periferal) disease treatment }(0.592)\end{array}$ \\
\hline 66 & Not studied & - & $\begin{array}{l}\text { Antineoplastic }(0.868) \\
\text { Antibacterial }(0.678)\end{array}$ \\
\hline 67 & Antifungal & Antifungal (0.690) & $\begin{array}{l}\text { Hepatic disorders treatment }(0.994) \\
\text { Hepatoprotectant }(0.786) \\
\text { Antiviral (arbovirus) }(0.713)\end{array}$ \\
\hline 68 & Antifungal & Antifungal (0.662) & $\begin{array}{l}\text { Hepatic disorders treatment }(0.987) \\
\text { Antineoplastic }(0.738)\end{array}$ \\
\hline 69 & Vasodilator Acyl CoA synthetase inhibitor & $\begin{array}{l}\text { Vasodilator }(0.881) \\
\text { Vasodilator, peripheral }(0.599)\end{array}$ & $\begin{array}{l}\text { Antieczematic }(0.830) \\
\text { Spasmolytic }(0.678)\end{array}$ \\
\hline 70 & Acyl CoA synthetase inhibitor & Vasodilator $(0.759)$ & $\begin{array}{l}\text { Spasmolytic }(0.649) \\
\text { Antineoplastic }(0.668)\end{array}$ \\
\hline 71 & Acyl CoA synthetase inhibitor & - & $\begin{array}{l}\text { Antieczematic }(0.917) \\
\text { Vasodilator }(0.901) \\
\text { Spasmolytic }(0.706)\end{array}$ \\
\hline 72 & Acyl CoA synthetase inhibitor & - & $\begin{array}{l}\text { Vasodilator }(0.881) \\
\text { Antieczematic }(0.830) \\
\text { Spasmolytic }(0.678)\end{array}$ \\
\hline
\end{tabular}


Table 5 continued

\begin{tabular}{llll}
\hline No. & Activity reviewed & Activities confirmed $(\mathrm{Pa})$ & Additional predicted activities $\left(\mathrm{Pa} \mathrm{a}^{\mathrm{a}}\right)$ \\
\hline $\mathbf{7 3}$ & Antimicrobial antiviral antineoplastic & Antineoplastic (0.409) & Antiischemic, cerebral $(0.752)$ \\
$\mathbf{7 4}$ & Antimicrobial antiviral antineoplastic & Antineoplastic (solid tumors) $(0.618)$ & Genital warts treatment $(0.656)$ \\
& & Antineoplastic (renal cancer) $(0.408)$ & Cytostatic $(0.562)$ \\
$\mathbf{7 5}$ & Antimicrobial antiviral antineoplastic & Antineoplastic (sarcoma) (0.482) & Gout treatment $(0.865)$ \\
& & & Genital warts treatment $(0.648)$ \\
$\mathbf{7 6}$ & Antimicrobial antiviral antineoplastic & - & Guanyl-specific ribonuclease T1 inhibitor (0.709) \\
& & & Genital warts treatment $(0.531)$ \\
$\mathbf{7 7}$ & Antimicrobial antiviral antineoplastic & Antineoplastic (sarcoma) $(0.469)$ & Anxiolytic $(0.896)$ \\
& & & Psychotropic $(0.745)$ \\
& & & Cognition disorders treatment $(0.608)$
\end{tabular}

${ }^{a}$ Only activities with $\mathrm{Pa}>0.5$ are shown

in Fig. 1. Elaiomycins K (16), L (17) and amide elaiomycin $\mathrm{K}$ (18), azoxy-type antibiotics, were detected in the culture filtrate extract of Streptomyces sp. Tü 6399. Both metabolites show weak antibacterial activity against Bacillus subtilis and Staphylococcus lentus as well as against the phytophathogenic Xanthomonas campestris [39].

Nematocidal antibiotics, jietacins A (19) and B (20), isolated from the culture broth of a Streptomyces sp. [40, 41], exhibited 10 times higher activities against the pine wood nematode Bursaphelenchus hgnicolus in comparison to avermectin Bla, which is known to have a potent activity against various nematodes and which is used as a nematocidal agent in the veterinary field $[42,43]$.

Hydrazides, geralcin C (21) was isolated from Streptomyces sp. LMA-545 together with geralcins A, B, D and E. Geralcin $\mathrm{C}$ has exhibited an $\mathrm{IC}_{50}$ of $0.8 \mu \mathrm{M}$ against $\mathrm{KB}$ and HCT116 cancer cell lines. Furthermore, geralcin C inhibited the E. coli DnaG primase, a Gram-negative antimicrobial target, with an $\mathrm{IC}_{50}$ of $0.7 \mu \mathrm{M}$ [44]. The antibiotic propanosine $(\mathrm{K}-76,22)$, found in extracts of $\mathrm{Mi}$ cromonospora chalcea 671-AV2, has shown inhibitory activity against Valsa ceratosperma [45]. Predicted activities compounds (22-39) shown in Table 3 and the structures shown in Figs. 1 and 2.

Another antibiotic, lyophyllin (23) was isolated from the mushroom Lyophyllum shimeji and showed inhibitory activity at a concentration of $50 \mu \mathrm{g} / \mathrm{mL}$, inducing forebrain blisters within the cranial mesenchyme [46]. Two antibacterial and anti-tumoural agents, antibiotic DC1881A (24) and DC1881B (25) are produced by Streptomyces sp. DO-118 [47].

Two azoxy compounds, KA-7367A (26) and KA-7367B (27), which have antifungal activity, have been found in the culture broth of Streptomyces sp. (KC-7367, FERM BP1277) [48]. Compound KA-7367A (26) showed antifungal activity against Candida albicans, Aspergilus fumigatus,
Cryptococcus neoformans, Trichophyton mentagraphytes, and T. rubrum.

An antitumor antibiotic with a diazene N-oxide structure, calvatic acid (alvatic acid or calvatinic acid, 28), and a methyl derivative (29), are produced by the fungi Calvatia craniformis [49] and C. lilacina [50] and from puffball mushrooms Lycoperdon pyriforme [51]. Calvatic acid inhibited the growth of Gram-positive and Gram-negative bacteria at a concentration of 3-6 $\mu \mathrm{g} / \mathrm{mL}[50]$ and showed cytotoxic activity by inhibiting cultured Yoshida sarcoma cell growth [49], and it also displayed carcinostatic activity against hepatoma and K562 leukemia cells [52]. Calvatic acid also showed antibacterial activity against the Gramnegative, microaerophilic bacterium Helicobacter pylori [53]. Two calvatic acid analogues (30 and 31) have demonstrated anti-microtubular properties [54].

Azoformamide (32), its (E)-form (33 and 35), and its azoxy derivatives (34 and 36) were isolated from the puffball Lycoperdon pyriforme [55, 56]. Extracts of the basidiomycete Lycoperdon pyriforme yielded 4-methoxybenzene-1-azoformamide (33) and 4-methoxy-benzene-1ONN-azoxyformamide (34), which possess nematicidal activity against the parasitic nematode Meloidogyne incognita. The chlorinated derivative (37) is less active towards nematodes, but more cytotoxic compared to (33 and 34) [57]. Two azoxyformamides ( $\mathbf{3 4}$ and $\mathbf{3 6}$ ) and two azoformamide derivatives (38 and 39) were isolated from the fruiting bodies of Calvatia craniiformis and Lycoperdon hiemale, respectively. Compounds (34) and (39) showed radicle growth inhibitory activities against lettuce seedlings, suggesting that the azoxy moiety contributes to the inhibitory activity. The plant growth inhibitory activities of $(\mathbf{3 4}, \mathbf{3 6}$, and 39) against barnyard millet seedlings were also reported [58]. The red minor pigment deoxyrubroflavin (40, activity see in Table 4) was isolated from the pufball mushroom Calvatia rubro-flava [59]. The orange pigment rubroflavin (41) was found in the dried fruit bodies 

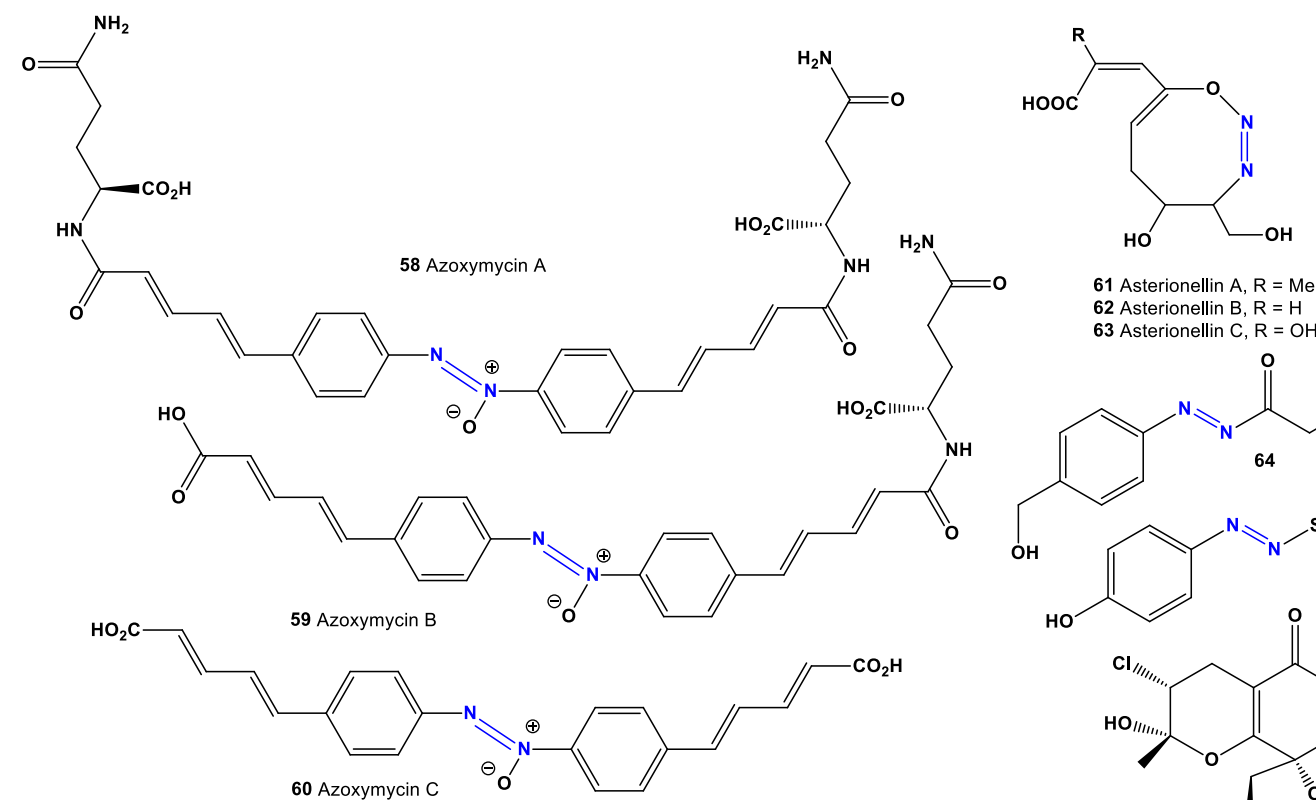

$$
\begin{aligned}
& 61 \text { Asterionellin } A, R=M e \\
& 62 \text { Asterionellin } B, R=H \\
& 63 \text { Asterionellin } C, R=O H
\end{aligned}
$$<smiles>Cc1ccc(/N=N/C(=O)CC[C@H](N)C(=O)O)cc1</smiles><smiles>CCC/C=C\C=C/C=C/C=N/N=N/O</smiles>

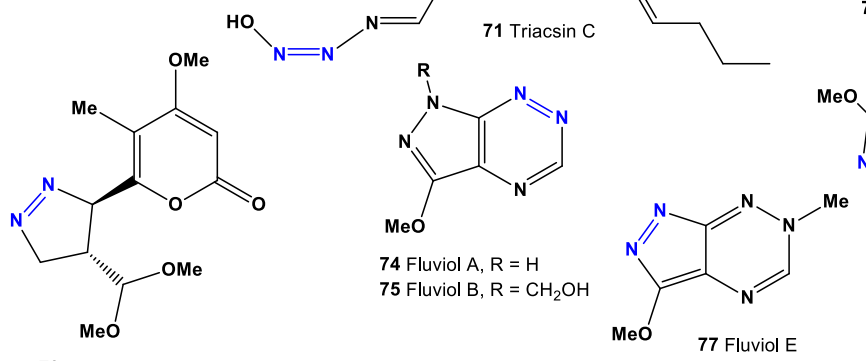<smiles>C/C=C\C=C/n1cnc2c(O)nnc-2n1</smiles>

73 Citreoazopyrone<smiles>Cc1cc2ccccc2nn1</smiles>

80 3-Me-Cinnoline<smiles>Cc1cnnc2ccccc12</smiles>

81 4-Me-Cinnoline<smiles>Oc1cccc2nnc3cccc(O)c3c12</smiles>

84<smiles></smiles>

82 4-Me-Cinnoline 2-Oxide<smiles>Cc1cn[n+]([O-])c2ccccc12</smiles>

3 4-Me-Cinnoline 1-Oxide<smiles>CCCCCCCCCCOS(=O)(=O)O</smiles>

HO

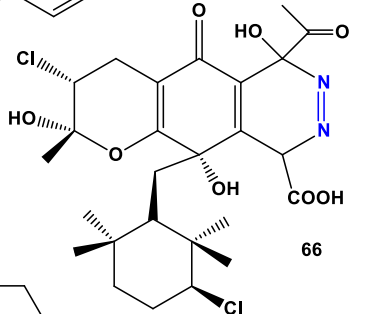<smiles></smiles>

$854849 \mathrm{E}$

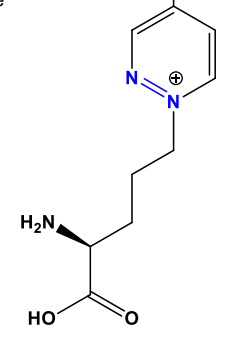

86 Pyridazomycin<smiles>COc1nn(C)c2nncnc12</smiles>

78 Pseudoiodinine<smiles>O=C1Nc2ccccc2C1=Cc1nnc2ccccc2c1C(=O)O</smiles><smiles>C=C(C)C(N)=O</smiles>
$\mathbf{c l}^{\ominus}$<smiles></smiles>

87

Fig. 3 Miscellaneous azo compounds produced by actinomycetes and fungal species 
Table 6 Confirmed and new biological activities of azo compounds (78-97) derived from actinomycetes and fungal species

\begin{tabular}{|c|c|c|c|}
\hline No. & Activity reviewed & Activities confirmed $(\mathrm{Pa})$ & Additional predicted activities $\left(\mathrm{Pa}^{\mathrm{a}}\right)$ \\
\hline 78 & Antimicrobial antiviral antineoplastic & Antineoplastic (sarcoma) (0.413) & $\begin{array}{l}\text { Atherosclerosis treatment }(0.924) \\
\text { Genital warts treatment }(0.600)\end{array}$ \\
\hline 79 & Cytotoxic antineoplastic & $\begin{array}{l}\text { Antineoplastic ( } 0.584) \\
\text { Antineoplastic (liver cancer) }(0.797)\end{array}$ & $\begin{array}{l}\text { Endothelial growth factor antagonist }(0.885) \\
\text { Angiogenesis inhibitor }(0.632)\end{array}$ \\
\hline 80 & Not studied & - & $\begin{array}{l}\text { Phobic disorders treatment }(0.728) \\
\text { Antineurotic }(0.685)\end{array}$ \\
\hline 81 & Not studied & - & $\begin{array}{l}\text { Antineurotic }(0.694) \\
\text { Phobic disorders treatment }(0.648)\end{array}$ \\
\hline 82 & Not studied & - & $\begin{array}{l}\text { Lysase stimulant }(0.787) \\
\text { Kidney function stimulant }(0.518)\end{array}$ \\
\hline 83 & Not studied & - & $\begin{array}{l}\text { Lysase stimulant }(0.787) \\
\text { Kidney function stimulant }(0.518)\end{array}$ \\
\hline 84 & Interleukin 4 antagonist & - & $\begin{array}{l}\text { Antiseborrheic }(0.815) \\
\text { Kidney function stimulant }(0.721) \\
\text { Phobic disorders treatment }(0.749)\end{array}$ \\
\hline 85 & Antibacterial & - & $\begin{array}{l}\text { Antineurotic }(0.806) \\
\text { Phobic disorders treatment }(0.752)\end{array}$ \\
\hline 86 & Not studied & - & Not predicted: MolCharge: 1 \\
\hline 87 & Not studied & - & Not predicted: MolCharge: 1 \\
\hline 88 & Cytotoxic & $\begin{array}{l}\text { Antineoplastic ( } 0.666) \\
\text { Antineoplastic (renal cancer) }(0.614)\end{array}$ & $\begin{array}{l}\text { Pterin deaminase inhibitor }(0.989) \\
\text { Natural killer cell stimulant }(0.587)\end{array}$ \\
\hline 89 & Cytotoxic antifungal & - & $\begin{array}{l}\text { Antiallergic }(0.765) \\
\text { Cytostatic }(0.712) \\
\text { Erythropoiesis stimulant }(0.692)\end{array}$ \\
\hline 90 & Antineoplastic antileukemic & $\begin{array}{l}\text { Antineoplastic (0.749) } \\
\text { Antileukemic }(0.622)\end{array}$ & $\begin{array}{l}\text { Genital warts treatment }(0.936) \\
\text { DNA synthesis inhibitor }(0.825) \\
\text { Cytostatic }(0.701)\end{array}$ \\
\hline 91 & Antineoplastic antileukemic & $\begin{array}{l}\text { Antineoplastic }(0.752) \\
\text { Antileukemic }(0.634)\end{array}$ & $\begin{array}{l}\text { Antimetabolite }(0.938) \\
\text { DNA synthesis inhibitor }(0.926) \\
\text { Neuroprotector }(0.910)\end{array}$ \\
\hline 92 & Antibiotic & - & Antineoplastic (sarcoma) (0.730) \\
\hline 93 & Not studied & - & $\begin{array}{l}\text { Antineoplastic }(0.768) \\
\text { Antibacterial }(0.614) \\
\text { Antifungal }(0.592)\end{array}$ \\
\hline 94 & Antimicrobial antiviral antineoplastic & $\begin{array}{l}\text { Glycopeptide-like antibiotic }(0.627) \\
\text { Antineoplastic }(0.406)\end{array}$ & Analgesic $(0.637)$ \\
\hline 95 & Antimicrobial antiviral antineoplastic & $\begin{array}{l}\text { Glycopeptide-like Antibiotic }(0.714) \\
\text { Antineoplastic }(0.519) \\
\text { Antibacterial }(0.409)\end{array}$ & Analgesic (0.670) \\
\hline 96 & Antimicrobial antiviral antineoplastic & $\begin{array}{l}\text { Glycopeptide-like antibiotic }(0.625) \\
\text { Antineoplastic }(0.443)\end{array}$ & Analgesic (0.601) \\
\hline 97 & Antimicrobial antiviral antineoplastic & $\begin{array}{l}\text { Glycopeptide-like antibiotic }(0.713) \\
\text { Antineoplastic }(0.547) \\
\text { Antibacterial }(0.424)\end{array}$ & Analgesic $(0.641)$ \\
\hline
\end{tabular}

${ }^{a}$ Only activities with $\mathrm{Pa}>0.5$ are shown 


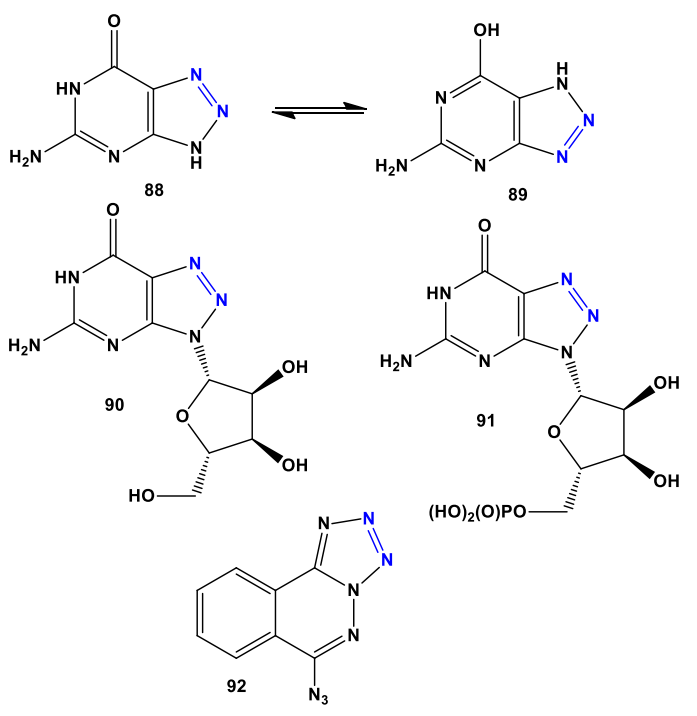

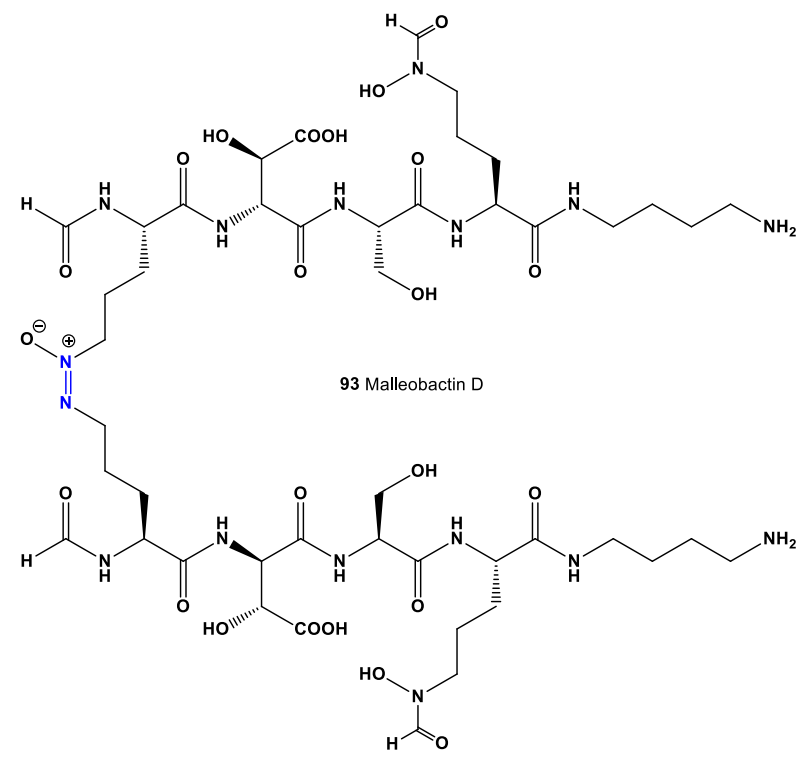

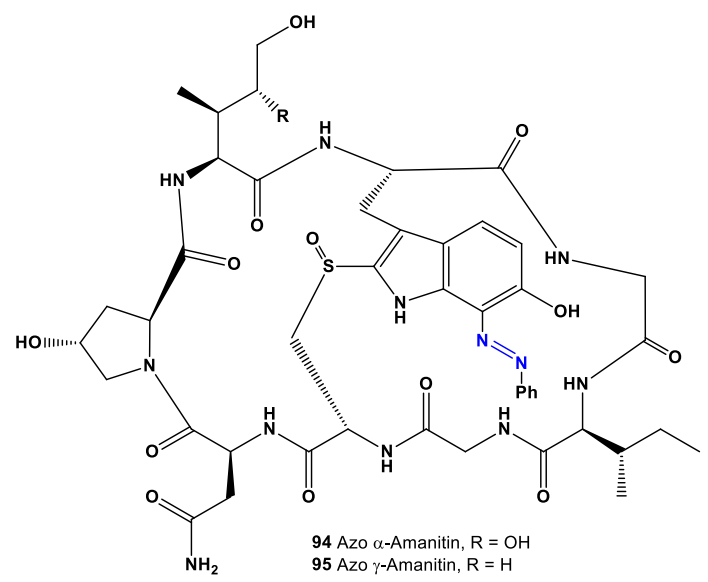

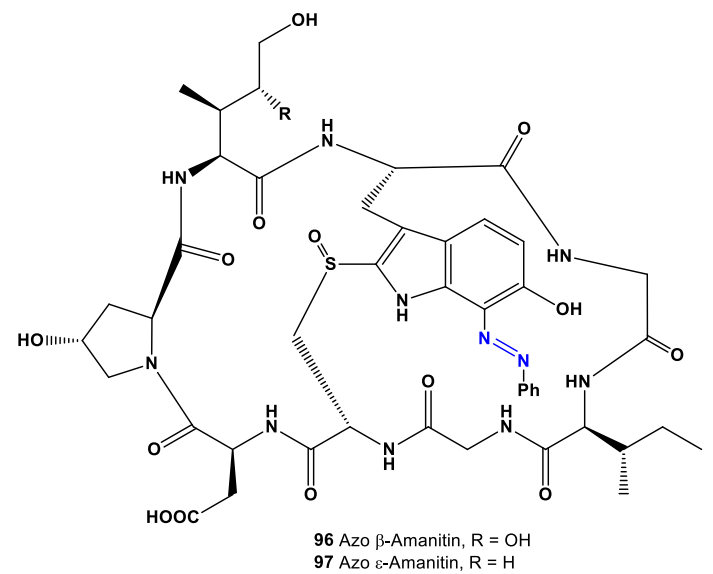

Fig. 4 Biological active triazole derivatives, siderophores and octapeptides derived from actinomycetes and fungal species

of North American puffball Calvatia rubro-flava and in $C$. craniformis [59, 60]. Oxyrubroflavin (42), craniformin (43), and cranformin (44) were isolated from C. rubro-flava $[59,60]$.

Two strains of the insect pathogenic fungus Entomophthora virulenta were found to produce a mixture of 4,4'-azoxybenzene dicarboxylic acid (45) and 4,4'-hydroxymethyl azoxybenzene carboxylic acid, which showed insecticidal activity (46) [61, 62]. A formazane derivative (47) has been isolated from Agaricus silvicola [63]. The mutagenic alkaloid necatorin $(\mathbf{4 8})$ has been isolated from the mushroom Lactarius necator [64-66]. Two azo dyes, 4,4'-dihydroxyazobenzene (49) and its methyl derivative (50), have been identified in the fresh sporophores of the mushroom Agaricus xanthodermus [67]. Several azo dyes are fungal toxins (51-57) and are produced by entomogenous fungi, such as Beauveria bassiana, Beauveria brongniartii, Metarhizium anisopliae, and Verticillium lecanii [66, 68-72].

Three novel aromatic azoxy compounds, azoxymycins A (58), B (59), and C (60), have been isolated and identified from Streptomyces chattanoogensis L10, and their biosynthetic pathways have been reported [73]. Predicted activities see in Table 5 and the structures shown in Figs. 2 and 3.

Asterionellins A (61), B (62), and C (63), eight membered compounds with an azoxy-like moiety, have been isolated from Asterionella sp. [74, 75]. The unstable agaritine derivative (64) and a metabolite (65) were extracted from the fruit-bodies of mushroom Agaricus xanthoderma [76]. Glutamylazophenol (62) was also found in Agaricus sp. [77]. Compound (65) has exhibited strong antibiotic and anticancer activities [77, 78]. 
Table 7 Confirmed and new biological activities of azo compounds (98-117) derived from plants

\begin{tabular}{|c|c|c|c|}
\hline No. & Activity reviewed & Activities confirmed $(\mathrm{Pa})$ & Additional predicted activities $\left(\mathrm{Pa}^{\mathrm{a}}\right)$ \\
\hline 98 & Cytotoxic & Antineoplastic (0.730) & $\begin{array}{l}\text { Antiprotozoal (plasmodium) }(0.641) \\
\text { Antiprotozoal }(0.579)\end{array}$ \\
\hline 99 & Immunosuppressant & - & $\begin{array}{l}\beta \text {-1,3-galactosyl-O-glycosyl-glycoprotein } \beta \text { - } \\
\text { 1,6-N-acetylglucosaminyl transferase } \\
\text { inhibitor }(0.954) \\
\text { Antineoplastic }(0.609)\end{array}$ \\
\hline 100 & Not studied & - & $\begin{array}{l}\text { Neurodegenerative diseases treatment }(0.920) \\
\text { Antiparkinsonian }(0.900) \\
\text { Anxiolytic }(0.796)\end{array}$ \\
\hline 101 & Not studied & - & $\begin{array}{l}\text { Acaricide }(0.721) \\
\text { Antiviral (arbovirus) }(0.681)\end{array}$ \\
\hline 102 & Not studied & - & Not predicted: MolCharge: 1 \\
\hline 103 & Not studied & - & Not predicted: MolCharge: 1 \\
\hline 104 & Not studied & - & $\begin{array}{l}\text { Antiinfertility, female }(0.940) \\
\text { Antineoplastic }(0.835) \\
\text { Phobic disorders treatment }(0.726)\end{array}$ \\
\hline 105 & $\begin{array}{l}\text { Toxic carcinogenic mutagenic } \\
\text { neurotoxic }\end{array}$ & $\begin{array}{l}\text { Carcinogenic }(0.975) \\
\text { Toxic }(0.932) \\
\text { Neurotoxic }(0.746)\end{array}$ & $\begin{array}{l}\text { Embryotoxic }(0.957) \\
\text { Teratogen }(0.952) \\
\text { Hepatotoxic }(0.716)\end{array}$ \\
\hline 106 & $\begin{array}{l}\text { Toxic carcinogenic mutagenic } \\
\text { neurotoxic }\end{array}$ & $\begin{array}{l}\text { Carcinogenic }(0.975) \\
\text { Toxic }(0.932) \\
\text { Eurotoxic }(0.746)\end{array}$ & $\begin{array}{l}\text { Embryotoxic }(0.957) \\
\text { Teratogen }(0.952) \\
\text { Hepatotoxic }(0.716)\end{array}$ \\
\hline 107 & Not studied & - & $\begin{array}{l}\text { Antineoplastic }(0.892) \\
\text { Genital warts treatment }(0.870) \\
\text { Antiinfective }(0.837)\end{array}$ \\
\hline 108 & $\begin{array}{l}\text { Toxic carcinogenic mutagenic } \\
\text { neurotoxic }\end{array}$ & $\begin{array}{l}\text { Carcinogenic }(0.964) \\
\text { Toxic }(0.943) \\
\text { Neurotoxic }(0.822)\end{array}$ & $\begin{array}{l}\text { Embryotoxic }(0.960) \\
\text { Teratogen }(0.950) \\
\text { Hematotoxic }(0.695)\end{array}$ \\
\hline 109 & Not studied & - & $\begin{array}{l}\text { Genital warts treatment }(0.876) \\
\text { Antineoplastic }(0.866) \\
\text { Vasoprotector }(0.851)\end{array}$ \\
\hline 110 & Not studied & - & $\begin{array}{l}\text { Antineoplastic }(0.892) \\
\text { Genital warts treatment }(0.870) \\
\text { Antiinfective }(0.837)\end{array}$ \\
\hline 111 & Not studied & - & $\begin{array}{l}\text { Antineoplastic }(0.892) \\
\text { Genital warts treatment }(0.870) \\
\text { Antiinfective }(0.837)\end{array}$ \\
\hline 112 & Not studied & - & $\begin{array}{l}\text { Antineoplastic }(0.897) \\
\text { Genital warts treatment }(0.870) \\
\text { Antiinfective }(0.837)\end{array}$ \\
\hline 113 & Not studied & - & $\begin{array}{l}\text { Antineoplastic }(0.889) \\
\text { Genital warts treatment }(0.857) \\
\text { Vasoprotector }(0.828)\end{array}$ \\
\hline 114 & Not studied & - & $\begin{array}{l}\text { Antineoplastic }(0.889) \\
\text { Genital warts treatment }(0.857) \\
\text { Vasoprotector }(0.828)\end{array}$ \\
\hline 115 & Not studied & - & $\begin{array}{l}\text { Antineoplastic }(0.900) \\
\text { Genital warts treatment }(0.864) \\
\text { Hepatic disorders treatment }(0.791)\end{array}$ \\
\hline
\end{tabular}


Table 7 continued

\begin{tabular}{llll}
\hline No. & Activity reviewed & Activities confirmed $(\mathrm{Pa})$ & Additional predicted activities $\left(\mathrm{Pa}^{\mathrm{a}}\right)$ \\
\hline $\mathbf{1 1 6}$ & Not studied & - & Antineoplastic $(0.889)$ \\
& & & Genital warts treatment $(0.857)$ \\
& Not studied & - & Vasoprotector $(0.828)$ \\
$\mathbf{1 1 7}$ & & & Antineoplastic $(0.889)$ \\
& & Genital warts treatment $(0.842)$ \\
& & Hepatic disorders treatment $(0.810)$ \\
\hline
\end{tabular}

${ }^{a}$ Only activities with $\mathrm{Pa}>0.5$ are shown

It is known that the anti-infective agent azamerone, a meroterpenoid, isolated from the saline culture of marinederived Streptomyces sp. Azamerone displays weak in vitro cytotoxicity against mouse splenocite populations of $\mathrm{T}$ cells and macrophages. A biosynthetic precursor azo compound (66) of azamerone has also been found in the same Streptomyces sp. [79]. The Streptomyces sp. Ank75 produced two azoxy antibiotics, 67 and $\mathbf{6 8}$, and both compounds exhibited antifungal activity against Candida albicans and Mucor miehei [80].

Two vasodilators, designated WS-1228 A (triacsin C, 69) and B (triacsin D, 70), were discovered in the culture of Streptomyces aureofaciens [81, 82]. Four years later, Omura and co-authors [83] reported two triacsins A (69) and B (70), inhibitors of acyl-CoA synthetase, which were isolated from the cultured broth of Streptomyces sp. The structurally related compounds WS-1228 A and B, known to be hypotensive vasodilators, were also found to inhibit acyl-CoA synthetase. The four compounds have N-hydroxytriazene moiety in their structures in common. The $\mathrm{IC}_{50}$ values for triacsin $\mathrm{A}$ and WS-1228 A were 5.5 and $3.6 \mu \mathrm{g} / \mathrm{mL}$, respectively. Triacsins A, B, C, and D, inhibitors of long chain acyl-CoA synthetase, possess different inhibitory potencies against the enzyme [84, 85]. AcylCoA synthetase activity in the membrane fraction of Raji cells was also inhibited by triacsins, which display the same hierarchy of inhibitory potency as that against the enzyme from other sources, that is, the inhibitory potency of triacsin C (71) is greater than that triacsin A, followed by that of triacsin $\mathrm{D}$ (72), and is greater than or equal to that of triacsin $\mathrm{B}$ [85].

A novel metabolite, citreoazopyrone (73), was isolated from the mycelium of Penicillium citreo-viride. It inhibited the growth of hypocotyls of lettuce seedlings [86]. A family of antibiotics named fluviols, which includes compounds $(\mathbf{7 4}, \mathbf{7 5}),(\mathbf{7 6}, \mathbf{7 7})$, and pseudoiodinine (78), are pyrazolo-[4,3-e]as-triazine derivatives, which are produced by Pseudomonas fluorescens var. pseudoiodinum and Nostoc spongiaeforme. All of these isolated compounds showed antimicrobial, antiviral, and antitumour activities [87-90]. Predicted activities compounds (78-98) shown in
Table 6 and the structures shown in Fig. 3. Schizocommunin (79) was isolated from a culture of the fungus Schizophyllum commune and exhibited strong cytotoxicity against murine lymphoma cells [91].

3- and 4-methylcinnolines ( $\mathbf{8 0}$ and $\mathbf{8 1}$ ) were found in the volatile constituents of Hibiscus esculentus pods [92]. Azoxy compounds (82 and $\mathbf{8 3}$ ) were found in yeast extract [93]. The cinnoline derivatives $(\mathbf{8 4})$ and $4849 \mathrm{~F}(\mathbf{8 5})$ were isolated from a culture of Streptomyces sp. Compound (84) was shown to be an inhibitor of the IL-4 receptor, and alkaloid 4849F (85) has shown antibacterial activity [94]. Pyridazomycin (86), an antifungal antibiotic produced by Streptomyces violaceoniger sp. griseofuscus, inhibited the growth of Mucor hiemalis [95]. Pyridazomycin (86) and its analog (87), as chloride salts showed antimicrobial activity [96]. Compounds (88 or 89), also known as 8-azaguanine, is produced from guanine by Spteptomyces albus [97]. The cytotoxic effect of 8-azaguanine on the growth of carcinoma, sarcoma, osteogenic sarcoma, lymphosarcoma, and melanoma in animals was reported more than 65 years ago [98] (see Fig. 4).

Compound (89), also known as pathocidin, is an antifungal antibiotic that has been isolated from actinomycetes [99, 100] and inhibited the growth of many fungi, including Penicillium chrysogenum. 8-azaguanine-3N- $\beta$-D-ribofuranosyl (90) and 5'-phosphate-3N- $\beta$-D-ribofuranosyl (91) are known as natural metabolites and showed anticancer activity against L-1210 lymphoid leukemia and adenocarcinoma 755, among other activities [101]. A toxic red-tide dinoflagellate, Gymnodinium breve, produced the antibiotic 6-azidotetrazolo[5,1-a]phthalazine (92) [102].

The Burkholderia species secretes a variety of extracellular enzymes with proteolytic, lipolytic, and hemolytic activities. Several strains also secrete toxins, antibiotics, and siderophores [103]. The unusual dimeric siderophore, malleobactin D (93), was isolated from Burkholderia pseudomallei [104].

The amatoxins are a group of bicyclic octapeptides produced by some species of mushrooms belonging to the Agaricales: Amanita phalloides, A. ocreata, A. verna, A. bisporigera, Conocybe filaris, Galerina marginata, $G$. 


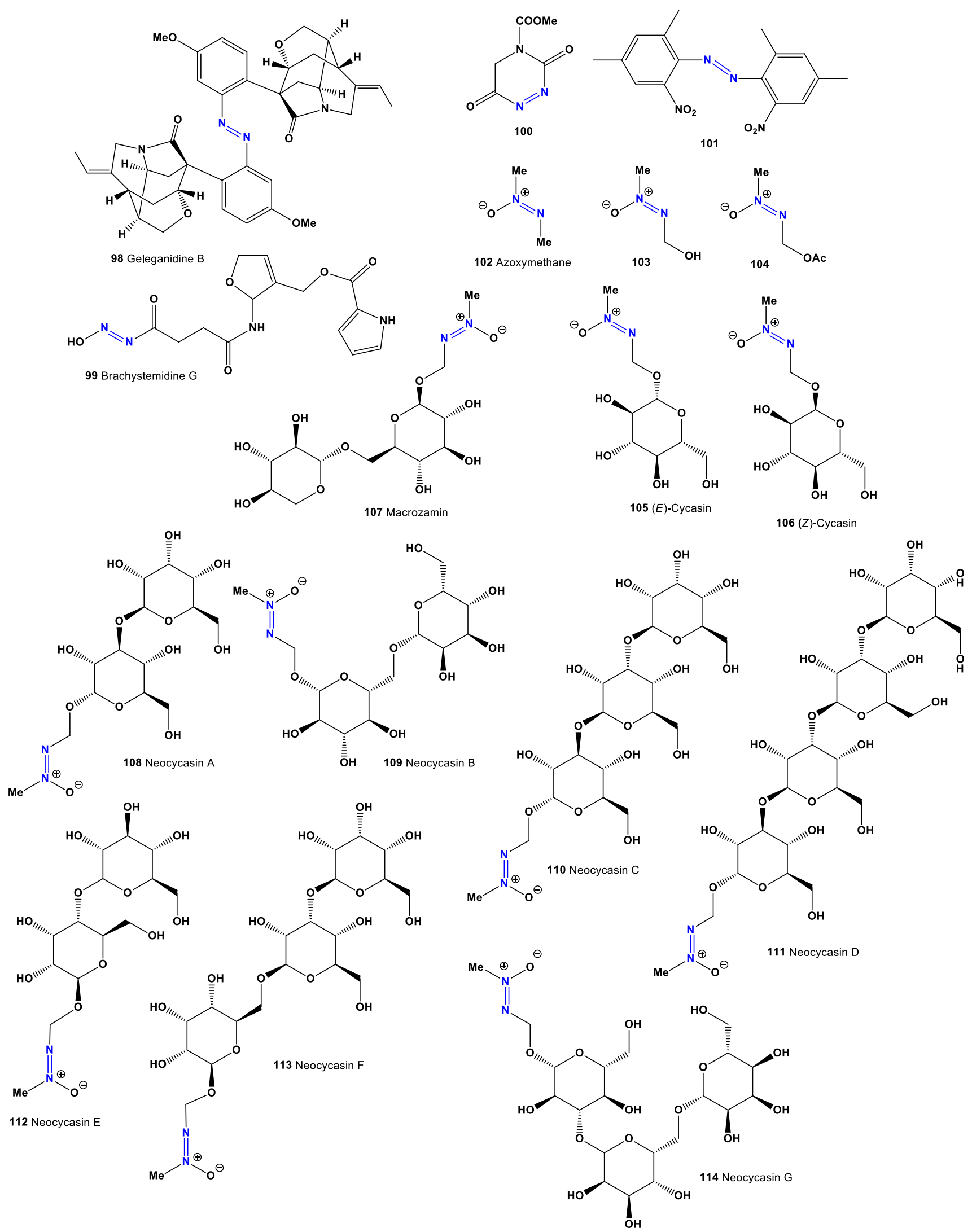

Fig. 5 Novel biological active azo compounds derived from plants 


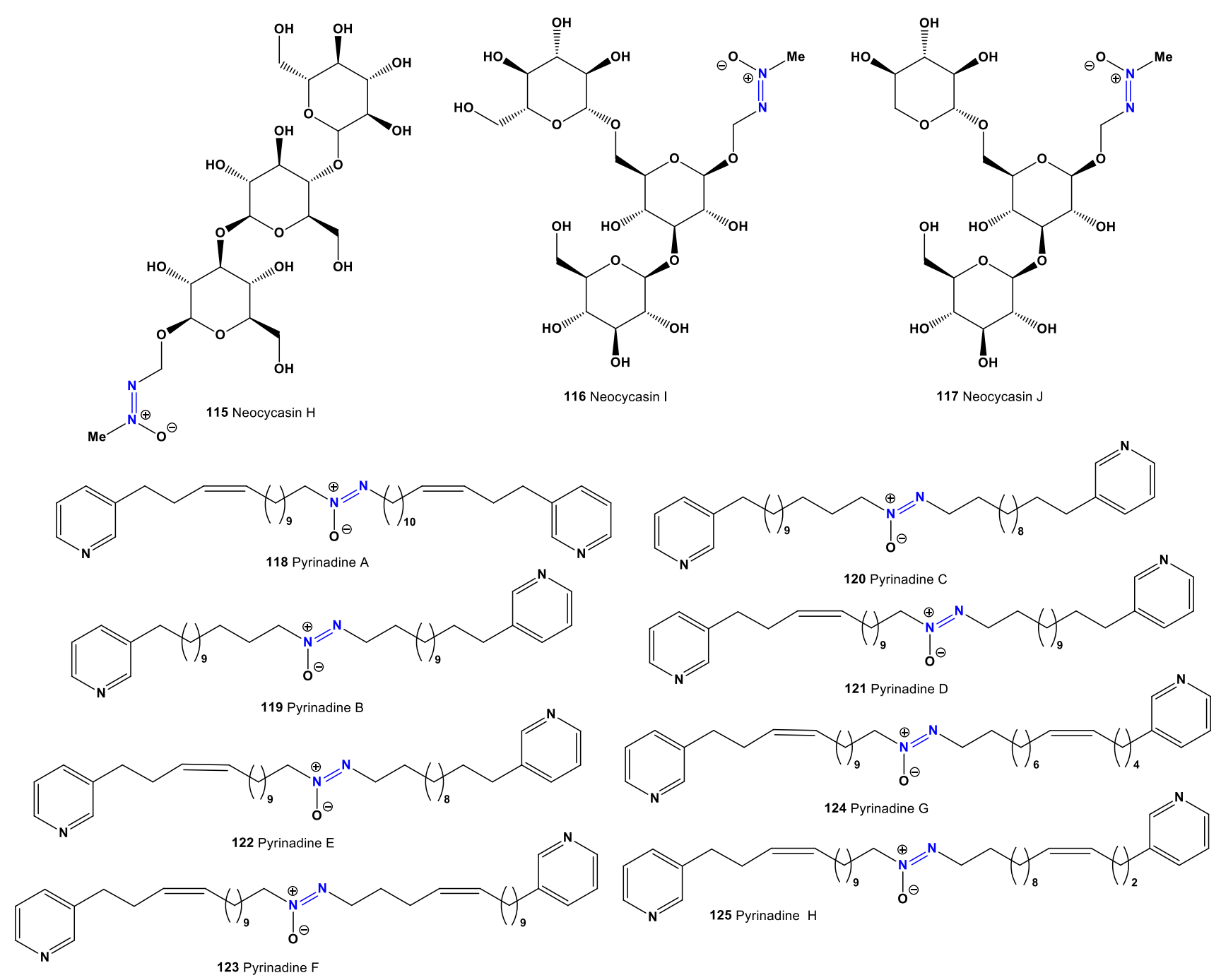

Fig. 6 Bioactive azoxy-glycosides derived from Cycadaceae plants and pyridine derivatives produced by marine sponge

venenata, Lepiotia castanea, $L$. helveola, $L$. subincarnata, L. brunneoincarnata, L. brunneolilacea, and close relatives. Selected amatoxins showed toxicity to heat, the digestive tract, and strong inhibition of RNA polymerase II [105-108]. Azo-amanitins (94-97) are semi-natural compounds, and they showed antiviral, antimicrobial, and anticancer activities [109-112]. Predicted activities compounds (88-97) shown in Table 6 and the structures shown in Fig. 3.

\section{Azo Metabolites Derived from Terrestrial and Marine Sources}

The novel dimeric monoterpenoid indole alkaloid, geleganidine D (98), was isolated from the roots of flowering plant Gelsemium elegans. It showed moderate cytotoxic activity against MCF-7 and PC-12 cells [113]. Predicted activities compounds (98-117) shown in Table 7 and the structures shown in Figs. 5 and 6.

Alkaloid brachystemidine G (99) was isolated from the roots of Brachystemma calycinum. This compound is a potent immunosuppressive agent, as demonstrated by its inhibition of mouse $\mathrm{T}$ and B-lymphocyte proliferation, with $\mathrm{IC}_{50}$ value of $5.6 \mu \mathrm{g} / \mathrm{mL}$ [114]. The 1,2,4-triazine derivative (100) was extracted from the seeds of the tropical flowering plant Butea monosperma [115]. It is known that the odor of this plant kills mosquitoes, the flowers are used as a dyeing color, and the gum, called kamarkas (Hindi), is used in food dishes [116]. Alkaloid (101) was isolated from the leaf extract of the flowering plant Aconnitum sungpanense [117].

Azoxy-glycosides have a common aglycone, methylazoxymethanol (MAM) and are found in Cycadaceae plants. To date, all of these glycosides that have been isolated have $\beta$-glycosidic linkages [118]. Methyl-azoxymethane (102), 
Table 8 Confirmed and new biological activities of azo compounds (118-125) derived from marine sponge

\begin{tabular}{|c|c|c|c|}
\hline No. & Activity reviewed & Activities confirmed $(\mathrm{Pa})$ & Additional predicted activities $\left(\mathrm{Pa}^{\mathrm{a}}\right)$ \\
\hline 118 & Cytotoxic & Antineoplastic (0.776) & $\begin{array}{l}\text { Antieczematic (0.693) } \\
\text { Antiinflammatory }(0.646)\end{array}$ \\
\hline 119 & Cytotoxic & Antineoplastic (0.747) & $\begin{array}{l}\text { Cardiovascular analeptic }(0.567) \\
\text { Fibrinolytic }(0.538)\end{array}$ \\
\hline 120 & Cytotoxic & Antineoplastic (0.747) & $\begin{array}{l}\text { Cardiovascular analeptic }(0.567) \\
\text { Fibrinolytic }(0.538)\end{array}$ \\
\hline 121 & Cytotoxic & Antineoplastic (0.771) & $\begin{array}{l}\text { Antieczematic }(0.671) \\
\text { Antiinflammatory }(0.645)\end{array}$ \\
\hline 122 & Cytotoxic & Antineoplastic (0.771) & $\begin{array}{l}\text { Antieczematic }(0.671) \\
\text { Antiinflammatory }(0.645)\end{array}$ \\
\hline 123 & Cytotoxic & Antineoplastic (0.771) & $\begin{array}{l}\text { Antieczematic }(0.671) \\
\text { Antiinflammatory }(0.645)\end{array}$ \\
\hline 124 & Cytotoxic & Antineoplastic (0.771) & $\begin{array}{l}\text { Antieczematic }(0.671) \\
\text { Antiinflammatory }(0.645)\end{array}$ \\
\hline 125 & Cytotoxic & Antineoplastic (0.776) & $\begin{array}{l}\text { Antieczematic }(0.693) \\
\text { Antiinflammatory }(0.646)\end{array}$ \\
\hline
\end{tabular}

${ }^{a}$ Only activities with $\mathrm{Pa}>0.5$ are shown

methylazoxy-methanol (MAM, 103), methylazoxymethanol acetate (104), and cycasin (105 and 106) metabolites were extracted from the seeds and roots of cycad plants Cycadaceae, Stangeriaceae, and Zamiaceae $[16,118-123]$, which are conifers common to the tropics and subtropics. MAM (103) was shown to induce a variety of tumors, primarily liver and renal cell carcinomas [124]. Cycasins (105 and 106) and macrozamin (107) are very toxic azoxyglycosides of Cycadales.

Azoxy-glycosides may have played an important ecological role as antiherbivore defenses. Cycasin, which together with macrozamin represent the major azoxy-glycosides occurring in cycads, has been reported to elicit responses similar to those that have been observed during carcinogenicity, mutagenicity, and neurotoxicity assays. The first isolation of a glycoside, neocycasin A (108), was reported [125]. More recently, a range of neocycasin compounds, including neocycasin B, C, D, E, F, G, H, I, and J (109-117), were isolated from different plants [126-133].

The first identified cytotoxic bis-3-alkylpyridine alkaloid containing an azoxy moiety, pyrinadine A (118), was isolated from an Okinawan marine sponge Cribrochalina sp. [134]. Additional cytotoxic bis-3-alkylpyridine alkaloids, pyrinadines B, C, D, E, F, G, and H (119-125) were isolated from the same Okinawan marine sponge. Pyrinodemins showed cytotoxicity against P388 murine leukemia cells $[135,136]$. Predicted activities compounds (118125) shown in Table 8 and the structures shown in Fig. 6.

\section{Concluding Remarks}

Natural azo metabolites comprise a rare group of natural products. They are primarily present in fungi, plant, and microorganisms have also been detected in some invertebrates. Little information is known about the biological activities of these metabolites. Nevertheless, reported activities for these isolated compounds have shown strong anticancer, antibacterial, antiviral, and other activities. The widest spectra of biological activities are exhibited by isolated azo metabolites. Natural azo compounds have been shown to be promising candidates for the development of new drugs used for the treatment of several diseases.

Acknowledgements The work was partially supported (TAG and VVP) in the framework of the Russian State Academies of Sciences Fundamental Research Program for 2013-2020.

\section{Compliance with Ethical Standards}

Conflict of interest The authors declare no competing financial interest.

Open Access This article is distributed under the terms of the Creative Commons Attribution 4.0 International License (http:// creativecommons.org/licenses/by/4.0/), which permits unrestricted use, distribution, and reproduction in any medium, provided you give appropriate credit to the original author(s) and the source, provide a link to the Creative Commons license, and indicate if changes were made. 


\section{References}

1. H.E. Bigelow, Chem. Rev. 9, 117-167 (1931)

2. T.A. La Rue, Lloydia 40, 307-321 (1977)

3. C.C. Nawrat, C.J. Moody, Nat. Prod. Rep. 28, 1426-1444 (2011)

4. L.M. Blair, J.J. Sperry, Nat. Prod. 76, 794-812 (2013)

5. V.M. Dembitsky, Phytomedicine 21, 1559-1581 (2014)

6. V.M. Dembitsky, J. Nat. Med. (Tokyo) 62, 1-33 (2008)

7. V.M. Dembitsky, Eur. J. Med. Chem. 43, 223-251 (2008)

8. F.M.D. Ismail, D.O. Levitsky, V.M. Dembitsky, Eur. J. Med. Chem. 44, 3373-3387 (2009)

9. D.M. Pereira, P. Valentão, P.B. Andrade, Dyes Pigm. 111, 124-134 (2014)

10. A.B. Soliev, K. Hosokawa, K. Enomoto, Evid. Based Complement. Altern. Med. 670349 (2011). doi:10.1155/2011/670349

11. V.M. Dembitsky, T. Řezanka, Folia Microbiol. 50, 363-391 (2005)

12. V.M. Dembitsky, Chem. Biodivers. 1, 673-781 (2004)

13. A. Sergeiko, V.V. Poroikov, L.O. Hanuš, V.M. Dembitsky, Open Med. Chem. J. 2, 26-37 (2008)

14. A. Torres, M. Hochberg, I. Pergament, R. Smoum, V. Niddam, V.M. Dembitsky, Eur. J. Biochem. 271, 780-784 (2004)

15. M. Wainwright, Biotech. Histochem. 85, 341-354 (2010)

16. A.M. Bode, Z. Dong, Cancer Prev. Res. (Phila.) 8, 1-8 (2015)

17. A.S. Shawali, N.A. Samy, J. Adv. Res. 6, 241-254 (2015)

18. V.M. Dembitsky, Tetrahedron 59, 4701-4720 (2003)

19. D.A. Filimonov, A.A. Lagunin, T.A. Gloriozova, A.V. Rudik, Chem. Heterocycl. Compd. 50, 444-457 (2014)

20. V.V. Poroikov, D.A. Filimonov, Y.V. Borodina, A.A. Lagunin, J. Chem. Inf. Comput. Sci. 40, 1349-1355 (2000)

21. V.M. Dembitsky, T. Gloriozova, V.V. Poroikov, Mini Rev. Med. Chem. 5, 319-336 (2005)

22. V.M. Dembitsky, T. Gloriozova, V.V. Poroikov, Mini Rev. Med. Chem. 7, 571-589 (2007)

23. V.M. Dembitsky, T. Gloriozova, V.V. Poroikov, Phytomedicine 22, 183-202 (2015)

24. D.O. Levitsky, T.A. Gloriozova, V.V. Poroikov, V.M. Dembitsky, Mathews J. Pharm. Sci. 1, 003 (2016)

25. M. Yamato, H. Iinuma, H. Naganawa, Y. Yamagishi, M. Hamada, T. Masuda, H. Umezawa, V. Abe, M. Hori, J. Antibiot. 39, 184-191 (1986)

26. Y. Ma, R.J. Parry, Microbiology 146, 345-352 (2000)

27. G. Bianchi, D. Dallavalle, L. Merlini, G. Nasini, S. Quaroni, Planta Med. 69, 574-576 (2003)

28. W.J. McGahren, M.P. Kunstmann, J. Am. Chem. Soc. 91, 2808-2810 (1969)

29. W.J. McGahren, M.P. Kunstmann, J. Am. Chem. Soc. 92, 1587-1590 (1970)

30. M. Nakayama, Y. Takahashi, H. Itoh, K. Kamiya, M. Shiratsuchi, G. Otani, J. Antibiot. 42, 1535-1540 (1989)

31. M. Fujiu, S. Sawairi, H. Shimada, H. Takaya, Y. Aoki, T. Okuda, K. Yokose, J. Antibiot. 47, 833-835 (1994)

32. Y. Aoki, M. Kondoh, M. Nakamura, T. Fujii, T. Yamazaki, H. Shimada, M. Arisawa, J. Antibiot. 47, 909-916 (1994)

33. T.H. Haskell, A. Ryder, Q.R. Bartz, Antibiot. Chemother. (Northfield) 4, 141-144 (1954)

34. J. Ehrlich, L.E. Anderson, G.L. Coffey, W.H. Feldman, Antibiot. Chemother. (Northfield) 4, 338-342 (1954)

35. L.E. Anderson, J. Ehrlich, S.H. Sun, P.R. Burkholder, Antibiot. Chemother. (Northfield) 6, 100-115 (1956)

36. K. Ohkuma, G. Nakamura, S. Yamashita, J. Antibiot. 10, 224-225 (1957)

37. L. Ding, B.S. Ndejouong, A. Maier, H.H. Fiebig, C. Hertweck, J. Nat. Prod. 75, 1729-1734 (2012)
38. N. Manderscheid, S.E. Helaly, A. Kulik, J. Wiese, J. Antibiot. 66, 85-88 (2013)

39. S. Omura, K. Otoguro, N. Imamura, H. Kuga, J. Antibiot. 40 , 623-629 (1987)

40. N. Imamura, H. Kuga, K. Otoguro, H. Tanaka, S. Omura, J. Antibiot. 42, 156-158 (1989)

41. K. Tsuzuki, F.S. Yan, K. Otoguro, S. Omura, J. Antibiot. 44, 774-784 (1991)

42. J.R. Egerton, D.A. Ostlind, L.S. Blair, C.H. Eary, Antimicrob. Agents Chemother. 15, 372-378 (1979)

43. A. Sugawara, M. Kubo, T. Nakashima, T. Hirose, Tetrahedron 71, 2149-2157 (2015)

44. G. Le Goff, M.T. Martin, B.I. Iorga, E. Adelin, J. Nat. Prod. 76, 142-149 (2013)

45. Y. Abe, J.I. Kadokura, A. Shimazu, H. Seto, N. Otake, Agric. Biol. Chem. 47, 2703-2705 (1983)

46. W.Y. Chan, T.B. Ng, J.S. Lam, J.H. Wong, Appl. Microbiol. Biotechnol. 85, 985-993 (2010)

47. H. Nakano, M. Hara, T. Katsuyama, Y. Uozaki, K. Gomi, U.S. Chem. Abstr. 119, 158353 (1993)

48. M. Nakaynma, H. Ito, I. Watanabe, M. Shiratsuchi, US Patent 4,981,954 (1991)

49. H. Umezawa, T. Takeuchi, H. Iinuma, M. Ito, M. Ishizuka, J. Antibiot. 28, 87-90 (1975)

50. R. Calvino, R. Fruttero, A. Gasco, A. Miglietta, L. Gabriel, J. Antibiot. 39, 864-868 (1986)

51. A. Gasco, A. Serafino, V. Mortarinin, E. Menziani, Tetrahedron Lett. 38, 3431-3432 (1974)

52. O. Brossa, E. Gadoni, A. Olivero, M. Seccia, A. Miglietta, L. Gabriel, E. Gravela, Res. Commun. Chem. Pathol. Pharmacol. 70, 143-153 (1990)

53. D. Boschi, C. Cena, R. Fruttero, M.I. Brenciaglia, Pharmazie 56, 670-672 (2001)

54. A. Miglietta, E. Gadoni, M. Buffa, A. Olivero, L. Gabriel, Eur. J. Drug Metab. Pharm. 20, 249-254 (1995)

55. T. Okuda, N. Nakayama, A. Fujiwara, Nippon Kingakukai Kaiho 23, 225-234 (1982)

56. T. Okuda, A. Fujiwara, Nippon Kingakkai Kaiho 23, 235-239 (1984)

57. B. Köpcke, A.A.H. Mayer, O. Sterner, Nat. Prod. Lett. 13, 41-46 (1999)

58. T. Kamo, M. Kashiwabara, K. Tanaka, S. Ando, H. Shibata, M. Hirota, Nat. Prod. Res. 20, 507-510 (2006)

59. Y. Takaishi, Y. Murakami, M. Uda, T. Ohashi, N. Hamamura, Phytochemistry 45, 997-1001 (1997)

60. F. Burkhard, A. Siegbert, W. Steglich, J. Fleischhauer, Eur. J. Org. Chem. 16, 3097-3104 (2001)

61. N. Claydon, J. Invertebr. Pathol. 32, 319-324 (1978)

62. D.W. Roberts, in Microbia Control of Pests and Plant Diseases 1970-1980, ed. by H.D. Burges (Academic Press, New York, 1981), pp. 441-464

63. W. Steglich, in Biologically Active Molecules (1989), pp. 1-8 64. T. Suortti, Food Chem. Toxicol. 22, 579-581 (1984)

65. T. Suortti, A. von Wright, J. Chromatogr. 255, 529-532 (1983)

66. S. Yannai, Dictionary of food compounds with CD-ROM: Additives, flavors, and ingredients (Chapman \& Hall/CRC, Boca Raton, 2004)

67. M. Gill, R.J. Strauch, Z. Naturforsch. C 39, 1027-1029 (1984)

68. A.T. Gillespie, N. Claydon, Pesticide Sci. 27, 203-215 (1989)

69. M.J. Bidochka, G.G. Khachatourians, J. Insect. Pathol. 58, 106-117 (1991)

70. G.G. Khachatourians, Biochemistry and molecular biology of entomopathogenic fungi, in The Mycota VI. Human and Animal Relationships, ed. by D.H. Howard, J.D. Miller (Springer, Berlin, 1996), pp. 331-363 
71. D.N. Pegler, Mushrooms and Toadstools (Mitchell Beazley, London, 1983)

72. C. Dickinson, J. Lucas, The Encyclopedia of Mushrooms (G. P. Putnam's Sons, New York, 1979)

73. Y.Y. Guo, H. Li, Z.X. Zhou, X.M. Mao, Org. Lett. 17, 6114-6117 (2015)

74. R. Wang, The investigation of biologically active secondary metabolites produced by diatoms. Ph.D. Dissertation, University of Rhode Island (1992)

75. Y. Shimizu, Chem. Rev. 93, 1685-1698 (1993)

76. B. Levenberg, J. Biol. Chem. 239, 2267-2274 (1964)

77. S. Hilbig, T. Andries, W. Steglich, T. Anke, Angew. Chem. 97, 1063-1069 (1985)

78. K. Dornberger, W. Ihn, W. Schade, D. Tresselt, A. Zureck, L. Radics, Tetrahedron Lett. 27, 559-566 (1986)

79. J.Y. Cho, H.C. Kwon, P.G. Williams, P.R. Jensen, W. Fenical, Org. Lett. 8, 2471-2474 (2006)

80. M. Bahi, Bandamycin as new antifungal agent and further secondary metabolites from terrestrial and marine microorganisms. Ph.D. Dissertation, Göttingen (2012)

81. K. Yoshida, M. Okamoto, K. Umehara, M. Iwami, J. Antibiot. 35, 151-156 (1982)

82. H. Tanaka, K. Yoshida, Y. Itoh, H. Imanaka, J. Antibiot. 35, 157-163 (1982)

83. S. Omura, H. Tomoda, Q.M. Xu, Y. Takahashi, Y. Iwai, J. Antibiot. 39, 1211-1218 (1986)

84. H. Tomoda, K. Igarashi, S. Omura, Biochim. Biophys. Acta 921, 595-598 (1987)

85. H. Ui, A. Ishiyama, H. Sekiguchi, M. Namatame, A. Nishihara, Y. Takahashi, K. Shiomi, K. Otoguro, S. Omura, J. Antibiot. 60, 220-222 (2007)

86. S. Kosemura, S. Yamamura, Tetrahedron Lett. 38, 3025-3026 (1997)

87. V.V. Smirnov, E.A. Kiprianova, A.D. Garagulya, S.E. Esipov, S.A. Dovjenko, FEMS Microbiol. Lett. 153, 357-361 (1997)

88. H.J. Lindner, G. Schaden, Chem. Ber. 105, 1949-1955 (1972)

89. K. Hirata, H. Nakagami, J. Takashina, T. Mahmud, M. Kobayashi, Heterocycles 43, 1513-1519 (1996)

90. M. Mojzych, J. Chem. Soc. Pak. 33, 698-702 (2011)

91. T. Hosoe, K. Nozawa, N. Kawahara, K. Fukushima, Mycopathologia 146, 9-12 (1999)

92. J.M. Ames, G. MacLeod, Phytochemistry 29, 1201-1207 (1990)

93. P.W. Brian, Bot. Rev. 17, 357-430 (1951)

94. K. Wang, L. Guo, Y. Zou, Y. Li, J. Wu, J. Antibiot. 60, 325-327 (2007)

95. R. Grote, Y. Chen, A. Zeeck, J. Antibiot. 41, 595-601 (1988)

96. H. Bockholt, J.M. Beale, J. Rohr, Angew. Chem. 106, 1733-1735 (1994)

97. K. Hirasawa, K. Isono, J. Antibiot. 31, 628-629 (1978)

98. K. Susugiura, G.H. Hitchings, L.F. Cavalieri, C.C. Stock, Cancer Res. 10, 178-185 (1950)

99. K. Anzai, J. Nagatsu, S. Suzuki, J. Antibiot. 14, 340-342 (1961)

100. K. Anzai, S. Suzuki, J. Antibiot. 14, 253 (1961)

101. A. Stachelska-Wierzchowska, J. Wierzchowski, A. Bzowska, B. Wielgus-Kutrowska, B Mol. 21, 44-49 (2016)

102. M.B. Hossain, D. van der Helm, Acta Crystallogr. 41C, 1199-1202 (1985)

103. V. Ludovic, M.C. Groleau, V. Dekimpe, E. Deziel, Prod. J. Microbiol. Biotechnol. 17, 1407-1429 (2007)

104. J. Franke, K. Ishida, M. Ishida-Ito, C. Hertweck, Angew. Chem. Int. Ed. 52, 8271-8275 (2013)

105. T. Wieland, H. Faulstich, C.R.C. Crit, Rev. Biochem. 5, 185-260 (1978)
106. F. Enjalbert, S. Rapiorm, J. Nouguier-Soulém, S. Guillon, N. Amouroux, C. Cabot, J. Toxicol. Clin. Toxicol. 40, 715-757 (2002)

107. C. Karlson-Stiber, H. Persson, Toxicon 42, 339-349 (2003)

108. J. Garcia, V.M. Costa, A. Carvalho, P. Baptista, P.G. de Pinho, M. de Lourdes Bastos, F. Carvalho, Food Chem. Toxicol. 86, 41-55 (2015)

109. E. Falck-Pedersen, P.W. Morris, D.L. Venton, Int. J. Peptide Protein Res. 21, 431-439 (1983)

110. E.S. Falck-Pedersen, Synthesis and characterization of $7^{\prime}$-azo- $\gamma$ amatoxins. Ph.D. Dissertation, University of Illinois. University Microfilms International, Ann Arbor, Michigan (1981)

111. V.M. Dembitsky, A.A.A. Al Quntar, M. Srebnik, Chem. Rev. 111, 209-237 (2011)

112. T. Wieland, Peptides of Poisonous Amanita Mushrooms (Springer, Berlin, 1986)

113. W. Zhang, X.J. Huang, S.-Y. Zhang, D.-M. Zhang, R.-W. Jiang, J.-Y. Hu, X.-Q. Zhang, L. Wang, W.-C. Ye, J. Nat. Prod. 78, 2036-2044 (2015)

114. Q. Lu, L. Zhang, G.-R. He, H.-X. Liang, G.-H. Du, Y.-X. Cheng, Chem. Biodivers. 4, 2948-2952 (2007)

115. M. Porwal, B.K. Mehta, D.N. Gupta, Nat. Acad. Sci. Lett. 2, 81-84 (1988)

116. F. Rana, M. Avijit, Int. J. Res. Pham. Chem. 2, 1035-1039 (2012)

117. X. Wang, Z. Lib, B. Yanga, Fitoterapia 75, 789-791 (2004)

118. P. Spencer, R.C. Fry, G.E. Kisby, Front. Genet. 3, 192-194 (2012)

119. G.L. Laqueur, M. Spatz, Cancer Res. 28, 2262-2267 (1968)

120. R.W. Morgan, G.R. Hoffmann, Mutat. Res. 114, 19-58 (1983)

121. D. Schneider, M. Wink, F. Sporer, P. Lounibos, Naturwis-senschaften 89, 281-294 (2002)

122. A. Prado, J. Ledezma, L. Cubilla-Rios, J.C. Bede, D.M. Windsor, J. Chem. Ecol. 37, 736-740 (2011)

123. S.F. Dossaji, Totins in certain indigenous Kenya plants. Ph.D. Thesis, University of Nairobi (1971)

124. T. Tanaka, H. Kohno, M. Murakami, R. Shimada, S. Kagami, Oncol. Rep. 7, 501-508 (2000)

125. K. Nishida, A. Kobayashi, T. Nagahama, T. Numata, Bull. Agric. Chem. Soc. Jpn. 23, 460-464 (1959)

126. T. Nagahama, T. Numata, K. Nishida, K. Agric, Chem. Soc. Jpn. 23, 556-559 (1959)

127. S.S. Chang, Y.L. Chan, M.L. Wu, J.F. Deng, T. Chiu, J. Toxicol. Clin. Toxicol. 42, 49-54 (2004)

128. F. Yagi, K. Tadera, Biochim. Biophys. Acta 1289, 315-321 (1996)

129. D.-F. Hwang, T.-Y. Chen, Ref. Modul. Food Sci. 326-330 (2016)

130. W.W. Wells, M.G. Yang, W. Bolzer, O. Mickelsen, Anal. Biochem. 25, 325-329 (1968)

131. A. Moretti, S. Sabato, G. Siniscalco, Phytochemistry 20, 1415-1416 (1981)

132. P. Lindblad, K. Tadera, F. Yagi, Environ. Exp. Bot. 30, 429-434 (1990)

133. J.E. Poulton, ACS Symp. Ser. 533, 170-190 (1993)

134. Y. Kariya, T. Kubota, J. Fromont, J. Kobayashi, Tetrahedron Lett. 47, 997-998 (2006)

135. Y. Kariya, T. Kubota, J. Fromont, J. Kobayashi, Bioorg. Med. Chem. 14, 8415-8419 (2006)

136. T. Kubota, K. Kura, J. Fromont, J. Kobayashi, Tetrahedron 69, 96-100 (2013) 